\title{
APPLICATION OF MEMS ENABLED EXCITATION AND DETECTION SCHEMES TO PHOTOACOUSTIC IMAGING
}

\author{
Jonas Kusch, Gordon M.H. Flockhart, Ralf Bauer and Deepak Uttamchandani \\ Centre for Microsystems \& Photonics, Department of Electronic \& Electrical Engineering \\ University of Strathclyde \\ Glasgow, UK
}

\begin{abstract}
This paper shows, for the first time, a MEMS enabled photoacoustic system, using a custom MEMS Q-switched $\mathrm{Nd}$ :YAG laser for generation of photoacoustic (PA) waves and a custom-built MEMS thin-film aluminium nitride piezoelectric micromachined ultrasound transducers for PA wave detection.
\end{abstract}

\section{KEYWORDS}

Photoacoustic, Imaging, MEMS, PMUT, Q-switched $\mathrm{Nd}$ :YAG, Miniaturisation, Aluminium Nitride

\section{INTRODUCTION}

Photoacoustic systems are of immense interest in the research fields of biomedical imaging and nondestructive testing applications as they can be used to scan through turbid media with improved specificity and resolution compared to conventional ultrasound (US) [1], [2]. A PA system consists of a pulsed laser and an US transducer to excite and detect PA waves, respectively. Size-reduction of PA systems using miniaturization of both the excitation and detection scheme, e.g. by using MEMS, can lead to more cost-effective PA sensing systems with improved sensitivity [3] and significantly wider application possibility.

During the past decade both electrical and optical MEMS based US sensors have become widely available. These include, amongst others, capacitive micromachined ultrasound transducers (CMUTs), piezoelectric micro-machined ultrasound transducers (PMUTs) and optically interrogated micro ring resonators (MRRs). CMUTs have been reported with centre frequencies of up to $29 \mathrm{MHz}$ at $100 \%$ relative bandwidth and offer high sensitivity [4], [5]. This is achieved by operating them at high bias voltages (of up to $100 \mathrm{~V}$ ) near or in collapsed mode, where the bias voltage is large enough to pull the top electrode partially into contact with the bottom electrode [6]. This operating mode puts the transducer at risk of failure and is problematic in biomedical applications due to the high voltage requirement. PMUTs use a membrane including a thin-film layer of piezoelectric material to detect US by deformation and deflection. The use of thin film materials and comparably simple manufacturing processes allows for higher degrees of flexibility with regards to transducer shapes. PMUTs are passive US sensors in that an external bias or supply voltage is not required for operation, and they achieve centre frequencies of $23.5 \mathrm{MHz}$ with comparably large relative bandwidths of $\leq 165 \%$ compared to CMUTs [7], [8]. Optical US sensors such as MRRs offer wide bandwidths ( $\geq 350 \mathrm{MHz}$ ) and high sensitivity, but suffer from higher costs due to optical components being more expensive and signal readout-schemes more elaborate [9][11].

Two types of pulsed laser sources are commonly used in PA systems. These are either solid-state Nd:YAG lasers producing laser pulses of short pulse widths $\tau$ ( $\tau \leq$ $10 \mathrm{~ns}$ ) and low pulse repetition frequencies (PRF) $\leq 20$ $\mathrm{Hz}$, or high power pulsed laser diodes (PLDs) with higher PRF, typically $5 \mathrm{kHz}-10 \mathrm{kHz}$, making them better suited to real-time PA imaging. However, PLDs typically produce pulses with longer $\tau(100 \mathrm{~ns} \leq \tau \leq 150 \mathrm{~ns})$ and lower pulse energy compared to solid-state lasers [12]. To improve the performance of PA systems, shorter laser pulse duration combined with higher PRF is still needed. We have previously reported a pulsed laser system based on a MEMS Q-switched Nd:YAG laser, which generated $34 \mathrm{~ns}$ pulses at $7.9 \mathrm{kHz}$ PRF with $7.9 \mu \mathrm{J}$ per pulse [13] This laser performance has the potential to meet the requirements of short pulse duration, sufficient energy per pulse and fast PRF requirements for real-time PA imaging.

This paper reports the utilization of this MEMS laser in a PA experiment. The paper also builds on the utilization of cost-effective, custom-built, thin-film aluminium nitride (AIN) PMUT arrays applied to photoacoustic scanning. While previous work used a 10 $\mathrm{Hz}$ PRF commercial Nd:YAG laser as the pulsed light source [15], here we use the MEMS laser as excitation source.

\section{SYSTEM}

\section{PA system}

The photoacoustic system (see Figure 1) is divided into an excitation part and a detection part. The excitation part consists of a custom MEMS Q-switched Nd:YAG laser using a diode side-pumped $\mathrm{Nd}$ :YAG crystal as laser gain medium. A cavity is created using a planar output coupler (reflectivity $\mathrm{R}=80 \%$ ) and a $700 \mu \mathrm{m}$ diameter gold-coated wide-angle MEMS scanning mirror $(R=96$ $\%$, radius of curvature of $0.4 \mathrm{~m}$ ). The scanning mirror is actuated using a $180 \mathrm{~V}_{\mathrm{PP}}$ square wave at $8.43 \mathrm{kHz}$. The mirror is periodically rotating in and out of optical alignment with the laser cavity, generating a Q-switched laser output with pulse widths of $\tau=50 \mathrm{~ns}$ and a maximum pulse energy of $23.8 \mu \mathrm{J}$ at a wavelength of $\lambda=$ $1064 \mathrm{~nm}$ at each passing through the cavity, resulting in a $P R F=8.43 \mathrm{kHz}$. The laser output is focussed onto a photoacoustic imaging target embedded in a gelatine $(8 \%$ $\mathrm{m} / \mathrm{m}$ ) phantom. The target is moved through the laser 


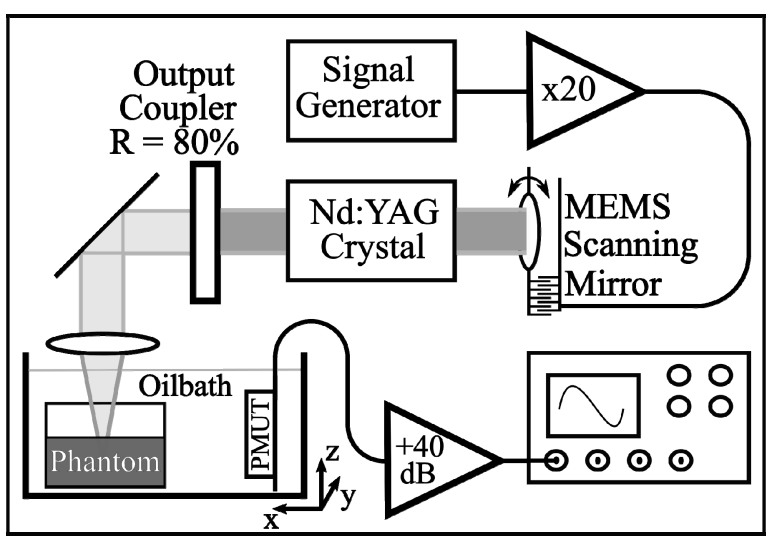

Figure 1: The PA setup consists of a MEMS $Q$ switched Nd:YAG laser as excitation source. The PMUT array, immersed in an oil bath, detects the generated $P A$ waves.

focal spot $(\mathrm{d}=20 \mu \mathrm{m})$ using a 3 -axis motorised stage (MTS50-Z8, Thorlabs). The generated photoacoustic wave is detected using a PMUT array. Both the PMUT array and the phantom are immersed in an oilbath for acoustic coupling. The PMUT output is amplified (Voltage Amplifier DHPVA, FEMTO Messtechnik $\mathrm{GmbH}$ ) and recorded using a high-speed DAQ card (National Instruments, NI PXIe-5122) at a sampling rate of $f_{S}=100 \mathrm{MSa} / \mathrm{s}$. The PXI chassis runs LabVIEW code to synchronise the sample positioning and data acquisition.

\section{Transducers}

In this paper two types of PMUT arrays are presented. Firstly, a single-type array of $8 \times 8$ membrane elements, each with a diameter of $240 \mu \mathrm{m}$ and a peak frequency measured to be $1.3 \mathrm{MHz}$ (see Figure 2, a). Secondly, a multi-type array with four subarrays of membrane transducers with diameters of $235 \mu \mathrm{m}, 310$ $\mu \mathrm{m}, 395 \mu \mathrm{m}$ and $500 \mu \mathrm{m}$ (see Figure 2, b). Their respective peak frequencies are measured to be $1.4 \mathrm{MHz}$, $0.8 \mathrm{MHz}, 0.55 \mathrm{MHz}$ and $0.25 \mathrm{MHz}$. The subarrays can be addressed individually. The arrays were characterised as described in [15].

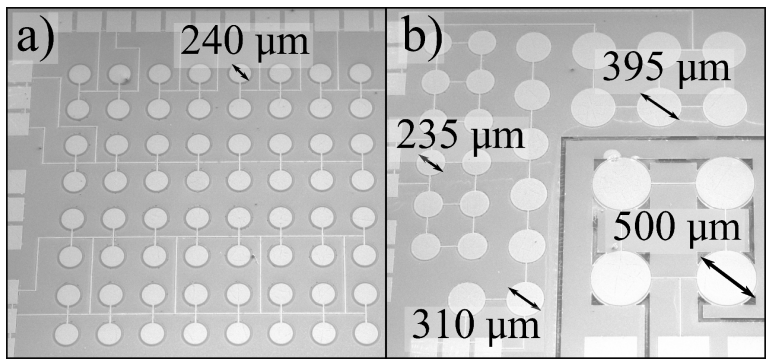

Figure 2: The single-type array (a) consists of 64 elements with a diameter of $240 \mu \mathrm{m}$. The multi-type array (b) consists of four sub-arrays of transducers with various diameters.

The arrays were produced using PiezoMUMPS, a cost-efficient multi-user MEMS process offered by MEMSCAP Inc, USA. Here, the piezoelectric material, a $500 \mathrm{~nm}$ thin-film (see Figure 3, grey) of AlN, is deposited between a layer of $\mathrm{Al}(1000 \mathrm{~nm})$ and $\mathrm{Cr}(20 \mathrm{~nm})$ and the doped SOI device layer (see Figure 3, red and orange, respectively). The former functions as top electrode while the latter is used as the bottom electrode. The membranes are then backside released from the $400 \mu \mathrm{m}$ Si handle wafer (see Figure 3, purple).

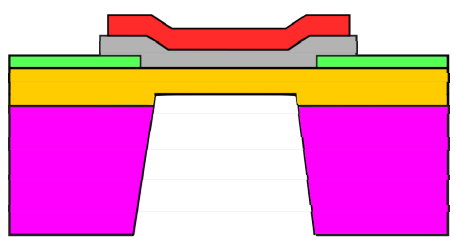
Aluminium $[1000 \mathrm{~nm}]$ $\square$ Aluminium Nitride $[500 \mathrm{~nm}]$ $\square$ Oxide Layer [200 nm] $\square$ Doped Silicon on Insulator (SOI) $[10 \mu \mathrm{m}]$ $\square$ Handle Wafer $[400 \mu \mathrm{m}]$

Figure 3: A schematic view of the cross-section of the membrane transducers and its various layers.

\section{METHODOLOGY}

Three kinds of target phantoms were scanned using the presented system. Firstly, a polyimide coated optical fibre with a diameter of $100 \mu \mathrm{m}$ embedded in a clear $8 \%$ $(\mathrm{m} / \mathrm{m})$ gelatine phantom was scanned using the full chip of the single-type and the multi-type arrays at step sizes of $\Delta x=10 \mu \mathrm{m}$ and $\Delta y=50 \mu \mathrm{m}$, in consecutive runs. This was done with a PRF of $8.43 \mathrm{kHz}$ and a pulse energy of $E=$ $1.27 \mu \mathrm{J}$ for the single-type array and a pulse energy of $E$ $=1.68 \mu \mathrm{J}$ for the multi-type array. At each step an averaged $(n=256)$ signal was recorded using the highspeed DAQ card. The power spectral density (PSD) was calculated using a Hamming window centred on the PA time-domain signal. The signal power was then calculated by integrating over the PSD in the limits of the PMUT array's bandwidth.

Secondly, a polyimide coated optical fibre embedded at a depth of $5 \mathrm{~mm}$ in a turbid $8 \%(\mathrm{~m} / \mathrm{m})$ gelatine phantom was scanned using the single-type array. The scattering property of the phantom was achieved by adding $3 \%$ $(\mathrm{m} / \mathrm{m})$ milk. The target was scanned using the single-type transducer with a laser pulse energy of $\mathrm{E}=3.47 \mu \mathrm{J}$ and sample position step sizes of $\Delta x=10 \mu \mathrm{m}$ and $\Delta y=50 \mu \mathrm{m}$. The signal power at each step was calculated as explained in the paragraph above.

Lastly, two ink-soaked (Drawing Ink: India Ink, Jackson's) suture threads (SILK USP 7/0 0.5, SMI) with a diameter of $60 \mu \mathrm{m}$ diameter arranged in a cross-pattern were embedded in a clear gelatine $(8 \% \mathrm{~m} / \mathrm{m})$ phantom. The target was scanned using the single-type PMUT array at a pulse energy of $E=12.6 \mu \mathrm{J}$ and step sizes of $\Delta x=30$ $\mu \mathrm{m}$ and $\Delta y=40 \mu \mathrm{m}$. The signal power for each pixel was calculated as the integral over the PSD.

\section{RESULTS}

The two-axis scan of the polyimide fibre in clear gelatine matrix using the single-type PMUT array is shown in Figure 4, a. The scanned fibre shows to have dimensions of $95 \mu \mathrm{m}$ in the $\mathrm{x}$ - direction and $750 \mu \mathrm{m}$ in the $z$ - direction. This elongation in the z-direction is due to the Rayleigh length of the focal spot of the laser, increasing the range of z-positions for which similar pulse energies are deposited onto the target. The time-domain signals are shown for a pixel in the centre of the target and for an off-target pixel (see Figure $4, b$, blue $\&$ red). The 

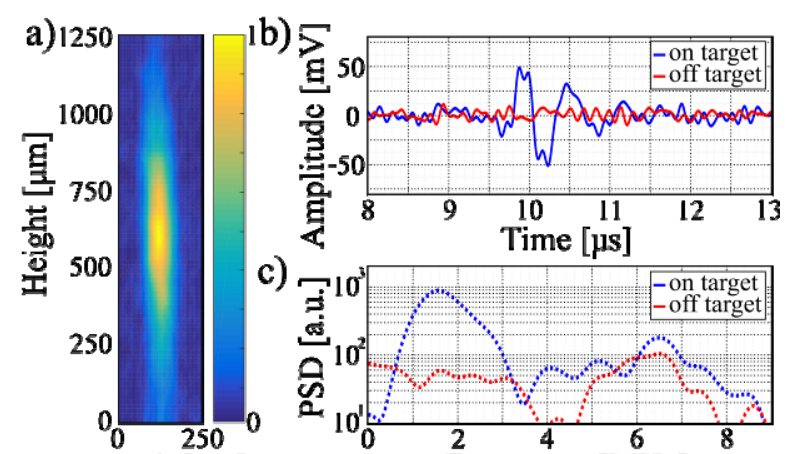

Length [ $\mathrm{\mu m}]$

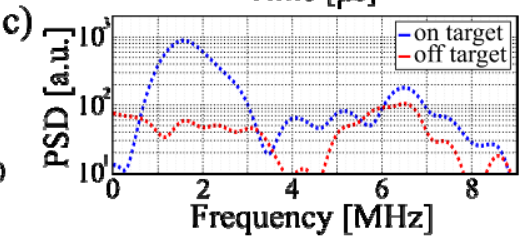

Figure 4: The scan of the target (a) using the singletype transducer array shows the target with dimensions of $95 \mu \mathrm{m}$ by $750 \mu \mathrm{m}$. The time domain trace (b) has an amplitude of $100 \mathrm{mV} V_{P P}$ and the PSD (c) shows the scan to have an SNR of 15.

resulting PSDs are shown in Figure 4, c. This shows the peak frequency at $1.5 \mathrm{MHz}$ with an SNR of 15 . The scan of the same target using the combined multi-type array shows to have dimensions of $130 \mu \mathrm{m}$ in the $\mathrm{x}$ - direction and $500 \mu \mathrm{m}$ in the $\mathrm{z}$ - direction. While the amplitude in the time-domain signal is higher compared to the single-type array $\left(150 \mathrm{mV}_{\mathrm{PP}}\right.$ to $100 \mathrm{mV}$ PP), so is the noise level (see Figure 5, b, blue \& red), resulting in an overall SNR of 10 (see Figure 5, c). The main peak is at $2.8 \mathrm{MHz}$, with side peaks at $1.5 \mathrm{MHz}$ and $4 \mathrm{MHz}$ (see Figure 5, c).
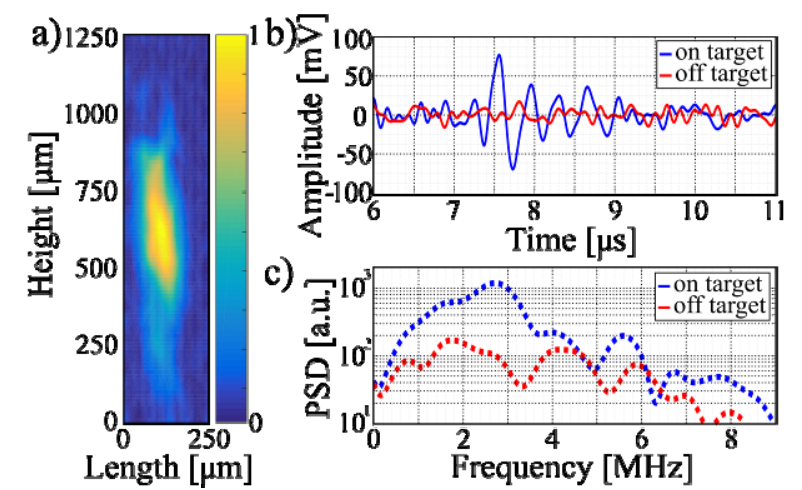

Figure 5: The scan of the target (a) using the multitype transducer array shows the target with dimensions of $130 \mu \mathrm{m}$ by $500 \mu \mathrm{m}$. The time domain trace (b) has an amplitude of $150 \mathrm{mV}_{P P}$ and the PSD (c) shows the scan to have an SNR of 10.

The scan of the polyimide fibre in the turbid gelatine phantom shows a diameter of $0.14 \mathrm{~mm}$ in the $\mathrm{x}$ - direction and $1.2 \mathrm{~mm}$ in the $\mathrm{z}$ - direction (see Figure 6, a). This broadening in both directions is caused by the broadening of the focal spot due to the higher scattering compared to the target scans in clear gelatine. The time-domain signal has an amplitude of $200 \mathrm{mV}_{\mathrm{PP}}$ (see Figure 6, b). This is a larger amplitude than shown in the previous experiments and is caused by the higher pulse energy used in this experiment. The PSD shows a peak frequency of $1.8 \mathrm{MHz}$ with an SNR of 50 (see Figure 6, c).

The scan of the two ink-soaked suture threads shows one at a higher intensity than the other (see Figure 7, a). This is caused by the former being closer to the focal plane than the other, with only data for one focal plane
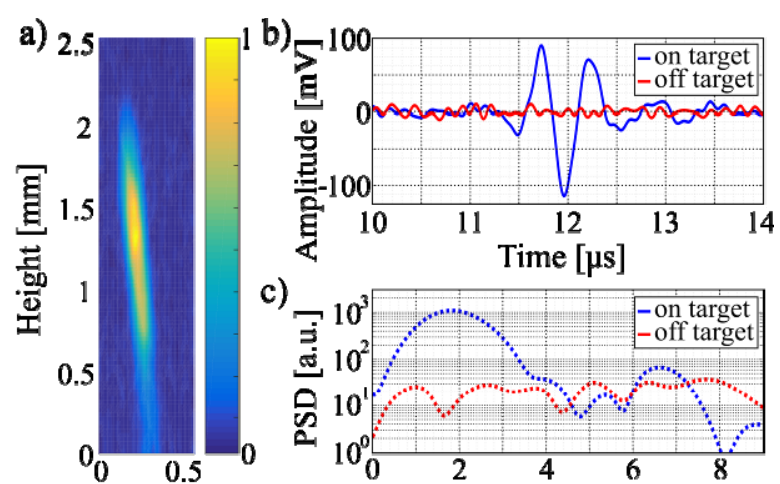

$$
\text { Length [mm] }
$$

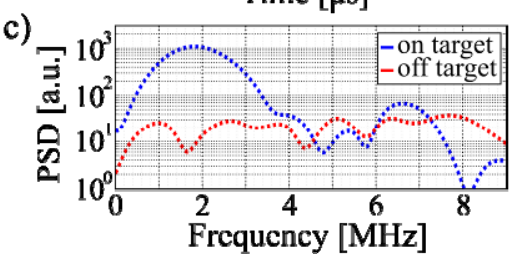

Figure 6: The scan of the target embedded in a turbid medium (a) using the single-type transducer array shows the target with dimensions of $0.14 \mathrm{~mm}$ by 1.2 $\mathrm{mm}$. The time domain trace (b) has an amplitude of $200 \mathrm{~m} V_{P P}$ and the PSD (c) shows the scan to have an SNR of 50 .

collected. Both show a diameter of $250 \mu \mathrm{m}$. This increase in diameter is caused by the carbon nanoparticles from the ink bleeding out into the surrounding matrix during the pouring of the top gelatine layer. The circular region of higher intensity around the cross-over point of the two threads is due to this effect as well. The signal trace of the top fibre has an amplitude of $275 \mathrm{mV}_{\mathrm{PP}}$, whereas the bottom fibre has an amplitude of $90 \mathrm{mV}_{\mathrm{PP}}$ (see Figure 7, b, red and blue, respectively). For both traces two peaks, at 1.2 $\mathrm{MHz}$ and at $4.7 \mathrm{MHz}$ can be seen in their respective PSDs (see Figure 7, c). Comparing these peaks to the noise floor (see Figure 7, c, green) SNRs of 30 and 70 can

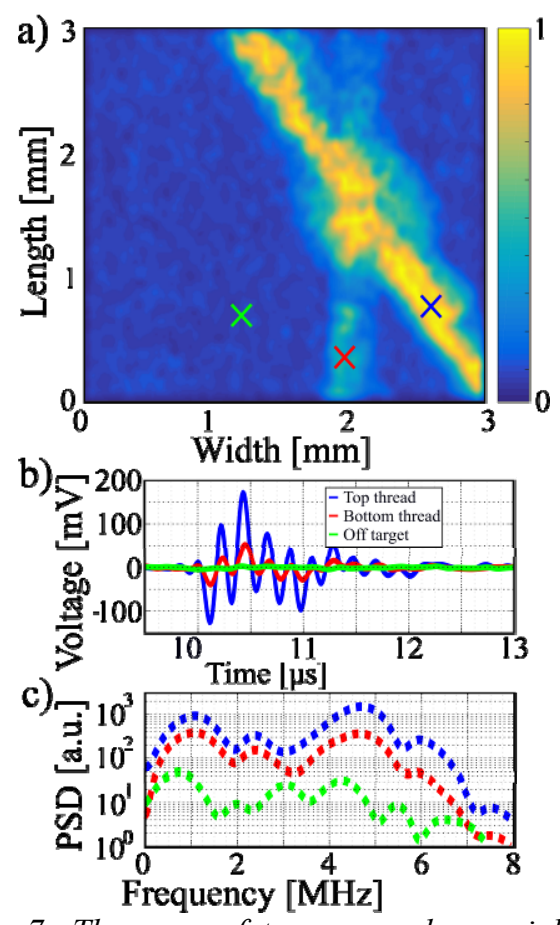

Figure 7: The scan of two crossed over ink-soaked suture threads (a) shows one at a higher intensity than the other. This can also be seen in the timedomain traces (b). Here, the top fibre (blue) has an amplitude of $275 \mathrm{mV} V_{P P}$ and the bottom fibre (red) has an amplitude of $90 \mathrm{mV} V_{P P}$. The PSD (c) shows peaks at 1.2 $\mathrm{MHz}$ and at $4.7 \mathrm{MHz}$. 
be calculated for the top fibre, for the $1.2 \mathrm{MHz}$ and the 4.7 $\mathrm{MHz}$ peak, respectively. For the bottom fibre the SNR for these two peaks is 17 and 12, respectively.

\section{DISCUSSION AND CONCLUSION}

The PSDs of the scans of the first phantom show peaks at $1.5 \mathrm{MHz}$ for the single-type array. This alteration compared to the $1.3 \mathrm{MHz}$ centre frequency from the characterisation was caused by variations in the transducer production process, as the PMUT array used in the characterisation was not the same chip as the one used in the experiments above. The main peak of the scan at $2.8 \mathrm{MHz}$ using the multi-type array is a superposition of the centre frequency of the $235 \mu \mathrm{m}$ sub-array and higher modes from the other sub-arrays. The peak at the centre frequency of the $235 \mu \mathrm{m}$ sub-array of $1.4 \mathrm{MHz}$ is also visible.

Scanning a polyimide coated fibre embedded in a turbid medium allows for higher pulse energies before ablation of the coating as the scattering causes the energy to be deposited over a larger area. This resulted in a 3-fold increase in SNR compared to clear gelatine. The elongation of the scanned image in $\mathrm{x}$ - and $\mathrm{z}$-direction compared to the clear gelatine scans is also due to the increase of the focal spot size and the Rayleigh length caused by scattering.

Higher pulse energies were used during the scans of the ink-soaked suture threads. This caused higher vibrational modes to be induced resulting in peaks at 4.7 $\mathrm{MHz}$ in addition to the $1.2 \mathrm{MHz}$ centre frequency peak.

In this paper, we have shown that PMUT arrays can be used in combination with a MEMS Q-switched $\mathrm{Nd}$ :YAG laser to scan targets in clear and turbid media. A future direction of this work is to concentrate on the digital signal processing and image processing which is needed to generate high quality images from the MEMS enabled PA system.

\section{REFERENCES}

[1] P. Beard, "Biomedical photoacoustic imaging," Interface Focus, vol. 1, no. June, pp. 602-631, 2011.

[2] H. Huan, A. Mandelis, B. Lashkari, and L. Liu, "Frequency-Domain Laser Ultrasound ( FDLU ) Non-destructive Evaluation of Stress - Strain Behavior in an Aluminum Alloy," Int. J. Thermophys., vol. 38, no. 4, pp. 1-11, 2017.

[3] R. Bogue, "Recent developments in MEMS sensors: a review of applications, markets and technologies," Sens. Rev., vol. 33, no. 4, pp. 300304, 2013.

[4] X. Zhang, F. Y. Yamaner, and O. Adelegan, "Design of High-Frequency Broadband CMUT Arrays," IEEE Int. Ultrason. Symp., pp. 1-4, 2015.

[5] D. F. Lemmerhirt, X. Cheng, R. D. White, C. A. Rich, M. Zhang, J. B. Fowlkes, and O. D. Kripfgans, "A 32 × 32 capacitive micromachined ultrasonic transducer array manufactured in standard CMOS," IEEE Trans. Ultrason. Ferroelectr. Freq. Control, vol. 59, no. 7, pp. 1521-1536, 2012.

[6] K. K. Park, Ö. Oralkan, and B. T. Khuri-Yakub, "A Comparison Between Conventional and Collapse-Mode Capacitive Micromachined Ultrasonic Transducers in 10-MHz 1-D Arrays," IEEE Trans. Ultrason. Ferroelectr. Freq. Control, vol. 60, no. 6, pp. 1245-1255, 2013.

[7] T. Pedersen, T. Zawada, K. Hansen, R. Loumoeller, and E. V Thomsen, "Fabrication of HighFrequency pMUT Arrays on Silicon Substrates," IEEE Trans. Ultrason. Ferroelectr. Freq. Control, vol. 57, no. 6, pp. 1470-1477, 2010.

[8] A. Hajati, D. Latev, D. Gardner, M. Ottosson, D. Imai, M. Torrey, M. Schoeppler, A. Hajati, D. Latev, D. Gardner, M. Ottosson, D. Imai, M. Torrey, and M. Schoeppler, "Monolithic ultrasonic integrated circuits based on micromachined semi-ellipsoidal piezoelectric domes," Appl. Phys. Lett., vol. 103, no. 202906, pp. 1-4, 2013.

[9] C. Zhang, S. Chen, T. Ling, L. J. Guo, and S. Member, "Review of Imprinted Polymer Microrings as Ultrasound Detectors: Design, Fabrication, and Characterization," IEEE Sens. J., vol. 15, no. 6, pp. 3241-3248, 2015.

[10] H. Li, B. Dong, Z. Zhang, H. F. Zhang, and C. Sun, "A transparent broadband ultrasonic detector based on an optical micro-ring resonator for photoacoustic microscopy," Sci. Rep., vol. 4, 2014.

[11] J. Yao and L. V Wang, "Sensitivity of photoacoustic microscopy," Photoacoustics, vol. 2, pp. 87-101, 2014.

[12] P. K. Upputuri and M. Pramanik, "Recent advances toward preclinical and clinical translation of photoacoustic tomography: a review," J. Bomedical Opt., vol. 22, no. 4, pp. 119, 2018.

[13] R. Bauer, W. Lubeigt, and D. Uttamchandani, "Dual Q -switched laser outputs from a single lasing medium using an intracavity MEMS micromirror array," Opt. Lett., vol. 37, no. 17, pp. 3567-3569, 2012.

[14] Q. Zhu, T. Chen, H. Liu, L. Sun, T. Wang, C. Lee, X. Le, and J. Xie, "An AlN - based Piezoelectric Micro - machined Ultrasonic Transducer ( pMUT ) Array," Proc. 16th Int. Conf. Nanotechnol. Sendai, Japan, August 22-25, 2016, pp. 731-734, 2016.

[15] J. Kusch, G. M. H. Flockhart, R. Bauer, and D. Uttamchandani, "Photoacoustic characterization of custom-made thin film AlN MEMS ultrasound transducers," in 2018 International Conference on Optical MEMS and Nanophotonics (OMN), 2018, vol. Lausanne, pp. 1-2. 